\title{
Atendimento em Reumatologia no Município de São Paulo: Análise na Gestão Plena do Sistema Único de Saúde (SUS) - Ano 2004
}

\section{Rheumatology Consultations in São Paulo City: Analyses of the Public Health Services (PHS) - Year 2004}

\author{
Deborah Colucci Cavalcante de Souza ${ }^{(1)}$, Catia Martinez Minto ${ }^{(2)}$, Rozana de Mesquita Ciconelli( ${ }^{(3)}$
}

\section{RESUMO}

Objetivo: avaliar quantitativamente os atendimentos ambulatoriais em reumatologia, no município de São Paulo, para a população usuária do SUS (Sistema Único de Saúde). Métodos: cálculo da porcentagem de execução da meta programada de consultas em reumatologia, conforme a Portaria 1101/GM, no município de São Paulo, para a população usuária do SUS. Resultados: a execução da meta programada em reumatologia mostrou-se satisfatória, considerando o município como um todo, porém, as porcentagens de execução da meta programada variaram extremamente em cada região da cidade. Das cinco regiões, apenas duas apresentaram cobertura satisfatória, com um número maior de consultas do que as programadas para a região. Nas outras três regiões, as porcentagens de execução de meta programada mostraram-se muito baixas. Conclusões: embora a oferta de consultas em reumatologia, no geral, tenha sido satisfatória, a distribuição deste recurso se mostrou extremamente heterogênea, sendo as regiões mais periféricas da cidade as menos favorecidas, por apresentarem um número menor de unidades de saúde que realizam tal atendimento. O excesso de consultas realizadas em relação às programadas pode ter como principais causas: o atendimento de uma demanda vinda de outros municípios, um número crescente de cidadãos tornando-se usuários do SUS e prováveis encaminhamentos inadequados para a especialidade.

Palavras-chave: consulta reumatológica, meta programada, município, SUS.

\section{INTRODUÇÃO}

O Sistema Único de Saúde (SUS) no Brasil representou um avanço na concepção de saúde, enquanto direito de todos e dever do Estado. Com essa nova maneira de administrar a saúde pública, passa a vigorar um sistema único, integrado e descentralizado, política e administrativamente, através da

\begin{abstract}
Objective: To evaluate the rheumatology outpatients, clinic consultations in the Public Health Services (PHS) in the city of São Paulo. Methods: Calculation of rate of the programmed rheumatologic consultations, according to $1101 \mathrm{G} / \mathrm{M}$ decree, in São Paulo, to the users of PHS. Results: The rate of the programmed rheumatologic consultations was satisfactory, taking into consideration the whole city, but it had an extreme range of variatins on each of the city regions. From the five regions, only two had a satisfactory cover, with a bigger number of consultations for these regions. The others three regions presented a very small execution percentage of the programmed goal. Conclusions: Although the availability of rheumatologic consultations, in general, was satisfactory, the distribution of this resource was extremely heterogeneous, being the suburbs, the less favorable regions because they have a smaller number of clinics than the other regions that carry out these consultations. The disproportionate number of the consultations in relation to the programmed ones might have its main causes due to: the treatment of people from other cities, a growing number of the citizens becoming PHS users and the probable unsuitable directions to a specialist.
\end{abstract}

Keywords: Rheumatologic consultation, programmed goal, city, Public Health Services.

municipalização dos serviços e ações de saúde.

Leis e normas foram, então, sendo criadas para assegurar a participação popular na questão saúde, como também para garantir o repasse dos recursos federais para os municípios.

No Quadro 1, apresentamos a evolução cronológica da criação de leis e normas em saúde, tanto em nível federal, como em nível municipal. ${ }^{(1,2,3,4)}$

Departamento de Medicina Interna, Disciplina de Reumatologia da Escola Paulista de Medicina da UNIFESP (Universidade Federal de São Paulo). Trabalho recebido em 22/09/05. Aprovado, após revisão, em 01/03/06.

1. Médica Reumatologista pós-graduanda (mestrado) da Disciplina de Reumatologia da Escola Paulista de Medicina da UNIFESP.

2. Assistente técnico da Coordenação de Integração e Regulação do Sistema de Saúde do Município de São Paulo.

3. Professora afiliada da Disciplina de Reumatologia da UNIFESP.

Endereço para correspondência: Rozana Mesquita Ciconelli, Rua Botucatu, 740, $3^{\circ}$ andar, Vila Clementino, São Paulo, CEP 04023-062, SP, Brasil, telefone: (11) 55796665, fax: (11) 5576-4239, e-mail: rozana@reumato.epm.br 
Quadro 1

Leis e Normas Federais e Municipais Relativas ao Sistema Único de Saúde (SUS)

\begin{tabular}{c|c|c}
\hline Ano & Nivel Federal & Nível Municipal \\
\hline $1987-1989$ & Instituição do SUS & SUDS e serviços municipais \\
1988 & & Municipalização de alguns equipamentos estaduais \\
$1989-1995$ & LOS (Leis Orgânicas da Saúde) \\
1990 & 8080 - Plano Municipal da Saúde é a base das atividades e da \\
programação de cada nível de direção do SUS & \\
19142 - Transferências intergovernamentais de recursos \\
financeiros na área da saúde \\
Norma Operacional Básica - 91 (NOB 91) \\
Adota a AIH (Autorização de Internação Hospitalar) e o SIH \\
(Sistema de Informação Hospitalar)
\end{tabular}

Norma Operacional Básica - 93 (NOB 93)

1993 Objetivos: Habilitação dos municípios nas categorias

Gestão Incipiente, Gestão Parcial ou Gestão Semiplena,

conforme o nível de complexidade

1994 Decreto Federal 1232 - Repasse dos recursos federais diretamente para os municípios

1995

Lei Municipal 11866 - Instituição do PAS (Plano de Atendimento à Saúde)

1996 Edição da NOB 96 - 0 município passa, de fato, a ser o responsável pelas demandas de saúde de seu território
A cidade é dividida em módulos assistenciais gerados por cooperativas privadas
NOAS 2001 - Norma Operacional de Assistência - objetiva regular a relação gestor-gestor e melhorar os processos de pactuação de referência, implementando a Pactuação Programada Integrada (PPI)*
Formação de 41 Distritos de Saúde.

Assinada a Gestão Plena da Assistência Básica, concretizada a partir de junho daquele ano
2002 NOAS 2002 - Regionalização da assistência à saúde, aprofundando a descentralização com eqüidade no acesso.
Divisão da cidade em 31 Coordenadorias de Saúde, correspondendo às 31 Sub-Prefeituras
Gestão Plena do SUS em São Paulo. A Secretaria Municipal de Saúde passa a ter a gestão de todos os serviços do SUS, inclusive dos prestadores privados credenciados ao SUS, efetivando-se as pactuações por meio das PPI

As Coordenadorias de Saúde são rearranjadas em número de cinco e passam a ser denominadas de Supervisão de Saúde

* PPI - Regulamentada pela Portaria MS/GM nº 483 de 06 de abril de 2001. Visa a integração das ações e serviços de assistência ambulatorial e hospitalar de complexidades básica, média e alta, orientando a organização do sistema de referência microrregional, regional e intermunicipal. 


\section{ACESSO AOS SERVIÇOS DE SAÚDE}

Antes de 2003, o acesso do usuário a consultas de especialidades era feito por meio de um sistema telefônico (0800), gerido pela Secretaria Estadual de Saúde. O próprio usuário fazia contato com uma central de marcação de consultas, o que possibilitava o acesso a partir de qualquer localidade do país. Com a Gestão Plena do SUS no município de São Paulo, em 2003, a Secretaria Municipal de Saúde passou por um processo de reorganização estrutural e funcional, implantando uma Coordenação de Regulação e Integração do Sistema de Saúde e criando as Centrais de Consultas de Especialidades, Regulação de Emergência, Leitos e Procedimentos de média e alta complexidades e instituindo os Fóruns de Pactuações entre as regiões do município, de modo a viabilizar uma rede de referência e contra-referência.

O antigo sistema 0800 foi então desativado pela Secretaria Estadual da Saúde e um novo foi implantado, o chamado Call Center, em que as unidades de saúde passariam a agendar as consultas de especialidades, e não mais o usuário.

A Secretaria Municipal de Saúde, através da Coordenação de Regulação e Integração do Sistema, iniciou uma forma de administração das ofertas de consultas de especialidades nos diversos serviços (municipais, estaduais, prestadores privados contratados e hospitais universitários), através de pactuações das ofertas, com lógica da necessidade. Estas pactuações são efetivadas periodicamente em Fóruns Regionais de Integração dos Serviços.

\section{ATENDIMENTOS AMBULATORIAIS EM REUMATOLOGIA NO MUNICÍPIO DE SÃO PAULO}

O objetivo do presente trabalho é avaliar quantitativamente a oferta de atendimentos ambulatoriais na especialidade de reumatologia, no município de São Paulo, através da análise da execução da meta programada, e, verificar a distribuição deste recurso na cidade para a população usuária do SUS.

Para avaliar os atendimentos na especialidade de reumatologia no município de São Paulo, analisamos o ano de 2004 .

NECESSIDADE DE CONSULTAS DE ESPECIALIDADE EM REUMATOLOGIA NO MUNICÍPIO DE SÃO PAULO

Para o cálculo da demanda de consultas de especialidade em reumatologia será considerada a população total estimada para o município de São Paulo e a população usuária do SUS, com base em série histórica.

A Portaria $n^{0} 1101 / G M$ de $12 / 06 / 2002^{(5)}$ estabelece os parâmetros de cobertura assistencial no âmbito do SUS e oferece subsídios para:

a) analisar a necessidade da oferta de serviços assistenciais à população;

b) auxiliar na elaboração do Planejamento e da Programação Pactuada e Integrada da Assistência à Saúde (PPI);

c) auxiliar no Acompanhamento, Controle, Avaliação e Auditoria dos serviços de saúde prestados no âmbito do SUS; Segundo a referida portaria, o cálculo do total de consultas médicas programadas (necessárias) para a cobertura assistencial ambulatorial subsidia o cálculo da necessidade de consultas de especialidades, e é realizado da seguinte maneira:

Necessidade de consultas médicas programadas total $(\mathrm{NTC})=2$ a 3 por habitante/ano

Do total das consultas médicas programadas, $62,7 \%$ são consultas de clínicas básicas (clínica médica, ginecologia/ obstetrícia e pediatria), $15 \%$ são referentes às consultas de urgência e emergência e $22,3 \%$ correspondem a consultas de especialidades.

As consultas programadas em reumatologia correspondem, aproximadamente, a 1,8\% das consultas de especialidades e perfazem $0,4 \%$ das NTC. Portanto:

Consultas de reumatologia $=$ NTC x $0,4 \%$ (parâmetro recomendado), onde NTC $=$ Número total de consultas programadas $=2$ a 3 por hab/ano.

Considerando a população do município de São Paulo, estimada em 2004, como 10.679.760 habitantes, temos como necessidade total de consultas médicas: $3 \mathrm{x}$ 10.679.760 hab $=32.039 .280$ consultas $/$ ano, portanto, NTC $=32.039 .280$ consultas/ano.

Consultas de reumatologia $=$ NTC $\times 0,4$ \% (parâmetro recomendado $)=32.039 .280$ consultas $/$ ano $\times 0,4 \%=$ 128.157 consultas/ano.

A Portaria $n^{0}$ 1101/GM define o número de internações hospitalares esperadas por clínica, porém considera para as especialidades um fator de ajuste que corresponde a todas as internações das especialidades não citadas na portaria. Portanto, há variação de acordo com cada realidade local, não viabilizando o uso de um parâmetro padrão para internações em cada especialidade.

\section{MATERIAL E MÉTODOS}

Para a pesquisa quantitativa das consultas realizadas pelas unidades prestadoras de serviços ao SUS foi utilizado o arquivo do DATASUS (Departamento de Informação e Informática do SUS).

Foi utilizada a porcentagem de execução da meta 
programada de consultas para avaliação da cobertura ambulatorial, em reumatologia, no município de São Paulo. A base para o cálculo da meta programada de consultas foi a Portaria 1101-GM/ 12.06.2002. ${ }^{(5)}$

\section{RESULTADOS}

\section{ATENDIMENTOS AMBULATORIAIS}

Um total de 31 Unidades Prestadoras de Serviços (UPS) realizou atendimento ambulatorial em reumatologia no município de São Paulo, no ano de 2004, com 123.929 con- sultas realizadas. Deste total de UPS, 8 são Ambulatórios de Especialidades (AE), 5 Unidades Básicas de Saúde/Centro de Saúde (UBS/CS), 11 Hospitais (sendo apenas 1 municipal), 3 NGA, I Centro de Referência do Idoso, I Sindicato (SECONCI - Sindicato da Indústria de Construção), l Centro de Reabilitação (Centro de Reabilitação Lar e Escola São Francisco) e 1 Associação Especializada (AACD - Associação de Apoio à Criança Defeituosa), segundo arquivo do DATASUS, conforme demonstra a Tabela 1. A Tabela 2 mostra as UPS por Região da cidade (correspondentes às Supervisões de Saúde), que prestaram atendimento

TABELA 1

Produção de consultas médicas na especialidade de reumatologia, nas Unidades Prestadoras de Serviços (UPS), no Municí́tio de São Paulo, janeiro a dezembro de 2004

\begin{tabular}{|c|c|c|c|c|c|c|c|c|c|c|c|c|c|}
\hline UPS & JAN & FEV & MAR & $\mathrm{ABR}$ & MAI & JUN & JUL & $\mathrm{AGO}$ & SET & OUT & NOV & DEZ & TOTAL \\
\hline UBS P. Manoel Nóbrega & - & - & - & - & - & - & - & - & 151 & 84 & - & 145 & 380 \\
\hline **UGA-I Hosp. Heliópolis & 780 & 1694 & 1052 & 1451 & 906 & 1320 & 1185 & 1363 & 1300 & 785 & 1343 & 1812 & 15.001 \\
\hline Sindicato - SECONCI - SP & 270 & 270 & 270 & 270 & 270 & 270 & 270 & - & 270 & 270 & - & 270 & 2.700 \\
\hline UBS Carandiru & - & - & - & - & - & - & - & - & - & 150 & - & 146 & 296 \\
\hline CS Dr. Victor A.H. Mello & 156 & 540 & 490 & 520 & 551 & 534 & 436 & 582 & 541 & 466 & 499 & 533 & 5.848 \\
\hline *NGA Várzea do Carmo & 928 & 1715 & 1307 & 1215 & 1563 & - & 1300 & 1299 & 1165 & 1174 & 827 & 506 & 12.999 \\
\hline Hosp Inf. Darcy Vargas & 26 & 40 & 35 & 32 & 42 & 35 & 37 & 42 & 36 & 49 & 57 & 10 & 441 \\
\hline Hosp. Sta Marcelina & 174 & 222 & 160 & 225 & 194 & 211 & 240 & - & 171 & 169 & - & 195 & 1.961 \\
\hline Hosp. São Paulo - UNID. I & 2500 & 2500 & 2500 & 2517 & 2505 & 2506 & 2499 & 2473 & 2513 & 2524 & 2513 & 2470 & 30.020 \\
\hline Hosp.Geral de S. Mateus & 1 & 232 & 217 & 227 & 203 & 236 & 195 & 279 & 235 & 196 & 213 & 286 & 2.520 \\
\hline Hosp. Ipiranga & 103 & 147 & 87 & 168 & 123 & 90 & 120 & 73 & 75 & 45 & 68 & 98 & 1.197 \\
\hline AACD & - & - & - & - & 9 & 9 & - & - & - & - & - & - & 18 \\
\hline Hosp.Geraldo Grajaú & 158 & 297 & 386 & 406 & 418 & 533 & 344 & 567 & 480 & 194 & 318 & 414 & 4.515 \\
\hline HC da FMUSP & 698 & 1571 & 1229 & 1302 & 1263 & 1185 & 1300 & 1501 & 1425 & 1327 & 1393 & 1702 & 15.896 \\
\hline Hosp.inf. mun.Men. Jesus & 41 & 115 & 75 & 83 & 95 & 83 & 65 & - & 115 & 66 & - & 45 & 783 \\
\hline Hosp. Regional Sul & 147 & 115 & 125 & 91 & 110 & 115 & 103 & 37 & - & - & - & - & 843 \\
\hline *NGA 8 - Pam Belém & 81 & - & 379 & 459 & 318 & 469 & 419 & 446 & 351 & 369 & 109 & - & 3.400 \\
\hline *NGA 39 - Santa Cruz & 43 & 92 & 70 & 98 & 88 & 108 & - & - & - & - & - & - & 499 \\
\hline Lar e Esc. S. Francisco & 42 & 111 & 111 & 111 & 111 & 97 & 90 & - & 90 & 68 & - & 54 & 885 \\
\hline AE J. Pirajussara & 178 & 336 & 235 & 257 & 268 & 269 & 280 & 352 & 307 & 297 & 315 & 70 & 3164 \\
\hline Centro Ref. Idoso & - & - & - & - & - & - & - & - & - & 104 & 112 & 154 & 370 \\
\hline Santa Casa de S.Paulo & 818 & 949 & 1041 & 928 & 1041 & 1041 & 1049 & 1041 & 520 & 520 & 508 & 420 & 9.876 \\
\hline AE Cecy K. Yasbek & 27 & 179 & 132 & 176 & 151 & 148 & 119 & - & 136 & 136 & - & - & 1.204 \\
\hline AE DST/AIDS V. Prudente & - & - & - & - & - & - & - & - & - & - & - & 80 & 80 \\
\hline AE Flavio Gianotti & 136 & 212 & 99 & 242 & 214 & 82 & 214 & - & 104 & 180 & - & 426 & 1.909 \\
\hline AE Freguesia do 0 & 226 & 314 & 246 & 541 & 538 & 267 & 137 & - & 242 & 188 & - & 219 & 2.918 \\
\hline AE J. Clíper & - & - & - & - & - & - & - & - & 38 & 140 & - & 151 & 329 \\
\hline AE Mooca-Ítalo Levocci & - & - & - & - & - & - & - & - & - & - & - & 72 & 72 \\
\hline AE Pq. da Lapa-Fernando R Cruz & 104 & - & 139 & 61 & 136 & 99 & 109 & - & 64 & - & - & - & 712 \\
\hline UBS Hermenegildo JR & 5 & - & 272 & 138 & 188 & 135 & 126 & - & 188 & 158 & - & 124 & 1.334 \\
\hline UBS J. Guairacá & 47 & 202 & 177 & 203 & 207 & 225 & 105 & - & 194 & 204 & - & 195 & 1.759 \\
\hline Total das UPS & 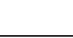 & & & & & & & & & & & & 123.929 \\
\hline
\end{tabular}


TABELA 2

Unidades Prestadoras de Serviços (UPS), por Região, QUe prestaram atendimento em Reumatologia (CONSUltas) No MUNicípIo de São PaUlo, ano 2004

\begin{tabular}{|c|c|}
\hline $\begin{array}{l}\text { Regiões da cidade } \\
\text { (correspondentes às cinco Supervisões de Saúde } \\
\text { do município de São Paulo) }\end{array}$ & UPS \\
\hline Leste & Hospital Santa Marcelina, Hospital Geral de São Mateus e Centro de Referência do Idoso \\
\hline Sudeste & $\begin{array}{c}\text { Hospital São Paulo/Unifesp/EPM, Hospital Ipiranga, Hospital Heliópolis, PAM Sta Cruz, } \\
\text { PAM Belém, AE Ceci Alex K. Yasbek, AE DST-AIDS V. Prudente, AE Flávio Gianotti, AE } \\
\text { Mooca, UBS J. Guairacá, UBS Hermenegildo M. Junior, UBS (AE) Pde. Manoel da Nóbrega, } \\
\text { AACD e Lar e Escola São Francisco }\end{array}$ \\
\hline Sul & Hospital Geral do Grajaú, Hospital Regional Sul, AE Pirajussara e AE Jd. Clíper \\
\hline Norte & AE Freguesia do Ó e UBS Carandiru \\
\hline Centro-0este & $\begin{array}{l}\text { Hospital das Clínicas da FMUSP, Hospital Infantil Menino Jesus, Hospital Darcy Vargas, } \\
\text { Santa Casa de São Paulo, PAM Várzea do Carmo,AE Parque da Lapa-Fernando R. Cruz e } \\
\text { SECONCI }\end{array}$ \\
\hline
\end{tabular}

Fonte - SIH/SUS, Datasus(Departamento de Informação e Informática do SUS).

ambulatorial em reumatologia, no ano de 2004.

A média mensal de atendimentos no município foi de 10.327 consultas/mês. Os atendimentos ambulatoriais em unidades hospitalares foram de 83.053 consultas e corresponderam a $67 \%$ do total realizado, sendo as demais unidades responsáveis por 40.876 atendimentos, 33\% do total de consultas do período. Quatro unidades hospitalares se destacaram em volume de consultas ambulatoriais realizadas: Hospital São Paulo da Escola Paulista de Medicina da Universidade Federal de São Paulo (UNIFESP)(24,22\% do total das consultas), Hospital das Clínicas, Instituto Central (12,83\%); Hospital SES Heliópolis (12,1\%) e Hospital Santa Casa de São Paulo (7,97\%), totalizando 57,12\% dos atendimentos realizados em ambulatórios ligados a hospitais do período. Do total de atendimentos realizados pelas 20 unidades ambulatoriais não ligadas a hospitais, duas foram responsáveis por quase metade dos atendimentos $(46 \%$ aproximadamente). São elas: CS SES Pinheiro "Victor A.H. de Mello" e NGA Várzea do Carmo.

\section{INTERNAÇÕES}

Conforme demonstrado na Tabela 3, ocorreram 661 internações em reumatologia no período sob análise em 35 hospitais. Observa-se que do total das 661 internações ocorridas neste ano quase metade, ou seja, 43,11\% foram no Instituto Central do Hospital das Clínicas ; 23\% no Hospital São Paulo da UNIFESP; 9,83\% no Hospital da Santa Casa de São Paulo. Os três Serviços juntos responderam por $75,94 \%$ do total das internações; as demais foram realizadas por outros 32 hospitais. A grande maioria destes 32 hospitais não possui Serviço de Reumatologia, portanto, as internações ocorreram esporadicamente.

As principais doenças reumáticas que justificaram as internações foram lúpus eritematoso sistêmico (CID M32) e artrite reumatóide (CID M05 e M06), com 385 e 132 internações respectivamente, visualizadas na Tabela 4 .

\section{CÁlCULO da PorCentagem de EXECUÇÃo dA META PROGRAMADA EM ATENDIMENTO AMBULATORIAL EM REUMATOLOGIA}

Embora a universalidade ao acesso seja uma das diretrizes do SUS, uma parcela da população não depende de seus serviços já que é usuária do sistema suplementar dos serviços de saúde. O município de São Paulo é uma das localidades com a mais alta proporção de população que possui convênios, seguros e planos de saúde. A contagem populacional de usuários do SUS é importante para planejamento e gestão de saúde pública. Este dado deve estar de acordo com a realidade da demanda pelos serviços de saúde e deve orientar e adequar a oferta dos mesmos. 
TABELA 3

Produção de internaÇões hospitalares por doenças reumáticas em Unidades Prestadoras de Serviços (UPS), no Município de São Paulo, janeiro a dezembro de 2004

\begin{tabular}{|c|c|c|c|c|c|c|c|c|c|c|c|c|c|}
\hline HOSP- CID - SP & JAN & FEV & MAR & $A B R$ & MAI & JUN & JUL & AGO & SET & OUT & NOV & DEZ & TOTAL \\
\hline Hosp. Mun. Inf. Menino Jesus & 1 & 2 & - & - & - & 1 & - & - & - & - & - & - & 4 \\
\hline Hosp. Mun. Jd. Ivaí & - & - & - & - & - & - & 1 & - & - & - & - & - & 1 \\
\hline Hosp. Mun. Jabaquara & 1 & - & - & - & - & - & - & - & 2 & - & - & - & 3 \\
\hline Hosp. Mun. Inacio Proença & - & - & - & - & - & 1 & - & - & - & - & - & - & 1 \\
\hline Hosp. Mun. V. Nhocuné & - & - & - & 1 & 1 & - & - & - & - & - & - & 2 & 4 \\
\hline Hosp. Mun. Planalto & - & - & 1 & - & - & - & - & - & 1 & - & - & - & 2 \\
\hline Hosp. Mun. Tide Setubal & - & - & 1 & - & - & - & - & - & - & - & - & - & 1 \\
\hline Hosp. Mun. Pirituba & - & 1 & - & - & - & 1 & - & - & - & - & 2 & - & 4 \\
\hline Hosp. Mun. Erm. Matarazzo & - & - & 1 & 1 & - & 2 & 1 & - & - & - & - & - & 5 \\
\hline Hosp. Mun. Tatuapé & - & 1 & - & 1 & - & - & - & - & - & - & - & - & 2 \\
\hline Hosp. SES Emílio Ribas & - & - & - & - & 2 & 1 & - & - & - & - & - & - & 3 \\
\hline Hosp. SES Candido Fontoura & - & - & - & - & - & - & - & - & - & - & - & 1 & 1 \\
\hline Hosp. SES Mandaqui & - & 1 & 1 & - & 1 & - & 1 & - & 2 & - & - & 1 & 7 \\
\hline Hosp. SES V.N. Cachoeirinha & - & - & - & - & - & - & - & 2 & 1 & 1 & - & - & 4 \\
\hline Hosp. SES de Guaianazes & - & - & - & 1 & - & - & - & - & - & - & - & 2 & 3 \\
\hline Hosp. SES de S. Mateus & 2 & 1 & - & - & - & 1 & - & - & - & - & - & - & 4 \\
\hline Hosp. SES Regional Sul & - & - & 1 & - & - & - & - & 1 & - & - & - & - & 2 \\
\hline Hosp. SES de V. Penteado & - & - & - & - & - & 1 & - & - & - & - & - & - & 1 \\
\hline Hosp. SES Brigadeiro & 1 & - & 1 & - & 1 & 1 & - & - & - & 1 & - & - & 5 \\
\hline Hosp. SES Heliópolis & 2 & 1 & 2 & 1 & 1 & 4 & & 4 & 3 & & 4 & 1 & 23 \\
\hline Hosp. SES Ipiranga & 1 & - & 1 & - & 1 & - & - & - & 3 & - & 1 & 1 & 8 \\
\hline Hosp. SES Darcy Vargas & - & - & 1 & 1 & - & - & - & - & - & - & 1 & - & 3 \\
\hline Hosp. SES Perola Bayington & - & - & - & - & 1 & - & - & - & - & - & - & - & 1 \\
\hline Hosp. SES Pedreira & - & - & - & - & - & - & - & - & - & - & - & 1 & 1 \\
\hline Hosp. SES do Itaim Paulista & - & - & - & - & - & 3 & - & 1 & - & - & - & - & 4 \\
\hline Hosp. SES do Grajaú & 1 & - & 1 & 2 & 1 & 2 & 2 & 1 & 2 & - & - & 2 & 14 \\
\hline Hosp. SES V. Alpina & - & - & - & - & - & - & - & - & - & - & - & 1 & 1 \\
\hline HC - FMUSP & 25 & 18 & 27 & 21 & 15 & 17 & 22 & 38 & 42 & 22 & 18 & 20 & 285 \\
\hline Hosp. Univ USP - SP & 2 & 2 & - & - & 1 & 1 & 1 & 2 & 2 & 2 & - & 1 & 14 \\
\hline Hosp. São Paulo-UNIFESP & 12 & 12 & 14 & 9 & 14 & 5 & 13 & 19 & 14 & 14 & 13 & 13 & 152 \\
\hline Hosp. Sta. Casa de São Paulo & 2 & 7 & 3 & 4 & 9 & 8 & 7 & 8 & 6 & 8 & 1 & 2 & 65 \\
\hline Hosp. Sta. Casa Sto. Amaro & - & - & - & - & - & - & - & 1 & - & - & - & - & 1 \\
\hline Hosp. da Benef. Portuguesa & 1 & - & 1 & - & - & - & - & 1 & 1 & 1 & - & 1 & 6 \\
\hline Hosp. Sta. Marcelina & 2 & - & - & 1 & 3 & 5 & 3 & 1 & 1 & 2 & 2 & 5 & 25 \\
\hline Hosp. Bandeirantes & - & - & - & - & - & - & - & - & - & - & - & 1 & 1 \\
\hline Total de Hospitais & & & & & & & & & & & & & 661 \\
\hline
\end{tabular}

Fonte - SIH/SUS, DATASUS (Departamento de Informação e Informática do SUS). 
TABELA 4

Produção de internações hospitalares, segundo doenças, no Município de São Paulo, nos meses de janeiro a DEZEMBRO DE 2004

\begin{tabular}{|c|c|c|c|c|c|c|c|c|c|c|c|c|c|}
\hline Doenças & JAN & FEV & MAR & $A B R$ & MAI & JUN & JUL & AG0 & SET & OUT & NOV & DEZ & TOTAL \\
\hline Lúpus eritematoso sistêmico & 32 & 27 & 33 & 27 & 30 & 30 & 27 & 39 & 46 & 34 & 24 & 36 & 385 \\
\hline Artrite reumatóide & 9 & 11 & 9 & 8 & 13 & 8 & 11 & 16 & 15 & 9 & 11 & 12 & 132 \\
\hline Dermatomiosite & 3 & 3 & 6 & 5 & 4 & 5 & 3 & 15 & 6 & 2 & 4 & 3 & 59 \\
\hline $\begin{array}{l}\text { Outras afecções do tecido } \\
\text { conjuntivo }\end{array}$ & 4 & 2 & 3 & 2 & - & 4 & 4 & 7 & 6 & 4 & 3 & 2 & 41 \\
\hline Artrite juvenil & 3 & 1 & 2 & 1 & 2 & 3 & 6 & 1 & 2 & 1 & - & - & 22 \\
\hline $\begin{array}{l}\text { Poliarterite nodosa e afecções } \\
\text { correlatas }\end{array}$ & 1 & 3 & 1 & - & - & 2 & - & 1 & 1 & - & - & 1 & 10 \\
\hline $\begin{array}{l}\text { Febre reumática sem menção } \\
\text { de comprometimento do } \\
\text { coração }\end{array}$ & - & 1 & - & - & 1 & 2 & - & - & 4 & - & - & 1 & 9 \\
\hline $\begin{array}{c}\text { Doenças sistêmicas do tecido } \\
\text { conjuntivo }\end{array}$ & - & - & 1 & - & - & - & - & - & - & 1 & - & - & 2 \\
\hline Coréia reumática & - & - & 1 & - & - & - & - & - & - & - & - & - & 1 \\
\hline $\begin{array}{l}\text { Total de doenças } \\
\text { internadas }\end{array}$ & & & & & & & & & & & & & 661 \\
\hline
\end{tabular}

Fonte - SIH/SUS, DATASUS (Departamento de Informação e Informática do SUS.

Segundo documento do CEINFO (Coordenação de Epidemiologia e Informação da Secretaria Municipal da Saúde) para consulta em planejamento em saúde ${ }^{(6)}$, a estimativa da população usuária do SUS varia de acordo com a região do município de São Paulo. Na Tabela 5, estão demonstradas as necessidades de consultas em reumatologia por região da cidade, as consultas realizadas por região e a porcentagem de execução de meta programada (E), calculada de acordo com a seguinte fórmula ${ }^{(5)}$ :

\section{$\mathrm{E}=\underline{\text { Total de procedimentos realizados }} \times 100$ Total de procedimentos programados}

\section{DISCUSSÃO}

Pelo demonstrado, constatamos que a cobertura ambulatorial no atendimento em reumatologia no município de São Paulo, medida pela porcentagem de meta executada, embora numericamente satisfatória, no geral, é extremamente desigual entre as regiões. Essa desigualdade se acentua quando comparamos as populações usuárias do SUS, sendo que nas regiões leste e norte menos de $30 \%$ dessa população dispõem das vagas necessárias na própria região. Das cinco regiões, apenas duas (sudeste e centro-oeste) apresentam produtividade satisfatória, sendo que nas três restantes (leste, sul e norte) o atendimento está bem abaixo do que seria o programado para a população da região. Na região sul, a disponibilidade de vagas, para a população usuária do SUS (entendida como consultas produzidas) para a especialidade é de $50 \%$, aproximadamente. Já nas regiões sudeste e centrooeste a disponibilidade de vagas é excessivamente maior do que a demanda regional (quatro e, praticamente, nove vezes a mais do que o necessário, respectivamente). Isto poderia explicar o deslocamento da população de outras regiões (leste, sul e norte) em busca de consultas nas regiões sudeste e centro-oeste. 
TABELA 5

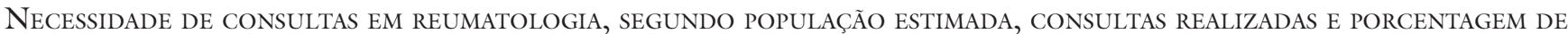
EXecução de meta Programada por Região da cidade de São Paulo, 2004

\begin{tabular}{|c|c|c|c|c|c|c|c|c|c|}
\hline $\begin{array}{l}\text { Regiões da } \\
\text { cidade* }^{*}\end{array}$ & Sub-Prefeituras & $\begin{array}{l}\text { População total } \\
\text { estimada }\end{array}$ & $\begin{array}{l}\text { População } \\
\text { usuária do } \\
\text { SUS }\end{array}$ & $\begin{array}{l}\text { Consultas } \\
\text { necessárias } \\
\text { População } \\
\text { total }\end{array}$ & $\begin{array}{l}\text { Consultas } \\
\text { necessárias } \\
\text { População } \\
\text { usuária do } \\
\text { SUS }\end{array}$ & $\begin{array}{l}\text { Consultas } \\
\text { realizadas }\end{array}$ & $\begin{array}{l}\text { Execução } \\
\text { da Meta } \\
\text { Programada } \\
\text { Pop. Total }\end{array}$ & $\begin{array}{c}\text { Execução } \\
\text { da Meta } \\
\text { Programada } \\
\text { Pop. usuária } \\
\text { do SUS }\end{array}$ & $\begin{array}{l}\text { No de UPS por } \\
\text { Região }\end{array}$ \\
\hline Leste & $\begin{array}{c}\text { Itaquera, } \\
\text { S.Miguel Paulista, } \\
\text { S.Mateus, Itaim, } \\
\text { Guaianazes, } \\
\text { Cid. Tiradentes } \\
\text { e Ermelino } \\
\text { Matarazzo }\end{array}$ & 2.396.940 hab. & $62,75 \%$ & 28.763 & 18.048 & 4.851 & $16,86 \%$ & $26,88 \%$ & $\begin{array}{l}\text { Dois hospitais } \\
\text { e } 1 \text { Centro de } \\
\text { Referência do } \\
\text { Idoso (total = 3) }\end{array}$ \\
\hline Sudeste & $\begin{array}{c}\text { Penha, } \\
\text { Aricanduva, } \\
\text { Mooca,Ipiranga, } \\
\text { V.Mariana, } \\
\text { Jabaquara e } \\
\text { V.Prudente/ } \\
\text { Sapopemba }\end{array}$ & 2.499.294 hab. & $47,29 \%$ & 29.992 & 14.182 & 57.758 & $192,58 \%$ & $407,26 \%$ & $\begin{array}{l}\text { Três Hospitais- } \\
\text { Escola, AACD, } \\
2 \text { PAM, } 4 \text { AE, } \\
3 \text { UBS e o } \\
\text { Lar Escola S. } \\
\text { Fancisco } \\
\text { (total }=14 \text { ) }\end{array}$ \\
\hline Sul & $\begin{array}{l}\text { Cid. Ademar, } \\
\text { M Boi Mirim, } \\
\text { Parelheiros, Santo } \\
\text { Amaro, Campo } \\
\text { Limpo e Socorro }\end{array}$ & 2.402.093 hab. & $57,49 \%$ & 28.825 & 16.701 & 8.851 & $30,70 \%$ & $52,99 \%$ & $\begin{array}{l}\text { Dois hospitais e } \\
2 \mathrm{AE}(\text { total }=4 \text { ) }\end{array}$ \\
\hline Norte & $\begin{array}{c}\text { Perus, Pirituba, } \\
\text { Freguesia do } \\
\text { Ó/Brasilândia, } \\
\text { Santana/Tucuruvi, } \\
\text { Casa Verde/ } \\
\text { Cachoeirinha, } \\
\text { Tremembé/ Jaçanã } \\
\text { e V. Maria/ } \\
\text { V. Gulherme }\end{array}$ & 2.136.977 hab. & $54,65 \%$ & 25.644 & 14.014 & 3.214 & $12,53 \%$ & $22,93 \%$ & $\begin{array}{c}1 \mathrm{UBS} \text { e } 1 \mathrm{AE} \\
(\text { total }=2 \text { ) }\end{array}$ \\
\hline $\begin{array}{l}\text { Centro- } \\
\text { Oeste }\end{array}$ & $\begin{array}{l}\text { Lapa, Butantã, } \\
\text { Pinheiros e Sé }\end{array}$ & 1.244.456 hab. & $38,34 \%$ & 14.933 & 5.725 & 49.255 & $329,84 \%$ & $860,35 \%$ & $\begin{array}{c}\text { Quatro } \\
\text { Hospitais-Escola, } \\
1 \text { Sindicato, } 1 \\
\text { CS, } 1 \text { PAM e } 1 \mathrm{AE} \\
(\text { total }=8)\end{array}$ \\
\hline $\begin{array}{l}\text { Todas as } \\
\text { Regiões }\end{array}$ & 31 Sub Prefeituras & 10.679.760 hab. & $53,52 \%$ & 128.157 & 68.589 & 123.929 & $96,70 \%$ & $180,68 \%$ & 31 UPS \\
\hline
\end{tabular}

Fonte dos dados populacionais - Estimativa da população exclusivamente usuária do SUS no Município de São Paulo, Documento da Secretaria Municipal da Saúde, São Paulo - 2004.

* Correspondentes às cinco Supervisões de Saúde. 
A distribuição das instituições de saúde na cidade é extremamente heterogênea, havendo região sem nenhum hospital para este tipo de atendimento, enquanto outras regiões da cidade concentram um grande número não só de hospitais como também de ambulatórios de especialidades.

Notamos que a região sudeste é a que concentra o maior número de instituições, sendo responsável por quase metade $(46,6 \%)$ do total das consultas realizadas. A região centro-oeste aparece com $39,74 \%$ do total das consultas. Juntas as regiões sudeste e centro-oeste realizaram $86,34 \%$ das consultas no ano de 2004. As outras três regiões (leste, norte e sul) realizaram apenas $13,66 \%$ do total. Constatamos, ainda, que poucas unidades ambulatoriais e hospitalares concentram a grande parte do atendimento em reumatologia no município de São Paulo. Observamos também que este atendimento tem se mantido constante, com uma média mensal de 10.327 consultas/mês no ano de 2004 .

Apesar da desigualdade de recursos regionais ser relevante, verifica-se, pelos dados apresentados, que o número de consultas efetivadas em reumatologia é, teoricamente, suficiente para dar cobertura não só à população usuária do SUS, mas praticamente à toda população do município (tx cobertura $=96,7 \%$ ). Esse número "excessivo" de consultas prestadas poderia ser explicado pelas seguintes possibilidades:

$\mathrm{I}^{\circ}$ ) usuários vindos de outros municípios são atendidos em São Paulo, aumentando assim a demanda neste município; tais consultas podem ser realizadas em instituições, em geral de ensino, que prestam o atendimento na especialidade tendo como critério somente o encaminhamento médico, sem a necessidade de passar pelo Sistema de Regulação; $2^{\circ}$ ) uma parcela da população não dependente do SUS pode estar utilizando os serviços. Essa possibilidade tem se mostrado bem factível, uma vez que parte da população que habitualmente fazia uso da medicina suplementar está, por questões financeiras, deixando de possuir esta forma de atendimento, o que provoca um conseqüente aumento no porcentual de usuários;

$3^{\circ}$ ) alguns planos de saúde não mantêm regularmente em seu quadro médico o especialista em reumatologia;

$4^{\circ}$ ) é possível que haja um "excesso" de encaminhamentos "desnecessários" do generalista para o especialista, ultrapassando a demanda teórica prevista de consultas;

$5^{\mathrm{O}}$ ) ainda podemos indagar se alguma parte de outros atendimentos especializados, como por exemplo, reabilitação, realizados em algumas instituições específicas, sejam, por vezes, lançados como consultas médicas de especialidade.

Em relação às internações hospitalares, verifica-se que os hospitais que mais internaram são as instituições de ensino $(75,94 \%$ do total): Instituto Central do Hospital das Clínicas, Hospital São Paulo da UNIFESP e Hospital Santa Casa de São Paulo.

É importante observar que o processo de atendimento ambulatorial é dinâmico, tendo em vista que novos médicos são contratados, outros se demitem e, portanto, o número de atendimentos em uma especialidade pode flutuar de um ano para outro.

Nos últimos 15 anos, houve apenas um concurso público na Secretaria Municipal de Saúde para médicos reumatologistas, realizado em 2004, em que foram nomeados 11 médicos, porém apenas 7 assumiram o cargo. Portanto, algumas unidades de saúde iniciaram o atendimento na especialidade de reumatologia apenas no final do ano de 2004.

Salientamos que os hospitais e ambulatórios não incluídos nesta análise compreendem àqueles que não se caracterizam como UPS ao SUS.

Pelo exposto, concluímos que a distribuição desigual das unidades de saúde que prestam atendimento em reumatologia, nas várias regiões do município de São Paulo, dificulta o acesso às consultas, especialmente para os cidadãos de regiões menos favorecidas em relação ao número dessas unidades. Essa dificuldade leva parte da população a procurar instituições de ensino, onde o atendimento acontece, geralmente, independentemente de agendamento prévio, o que explica, em parte, o excesso de atendimento ambulatorial nesta especialidade, apresentado por algumas dessas instituições e a conseqüente sobrecarga das mesmas.

Sugere-se ampliar o número de unidades de saúde que possam prestar atendimento em reumatologia nas regiões mais carentes deste atendimento, reorientando a demanda para suas próprias regiões e, desta forma, humanizando o atendimento.

Outra questão diretamente relacionada ao atendimento é a difícil fixação do profissional médico em regiões mais periféricas da cidade, pois sabemos que as demissões médicas nestes locais são muito mais freqüentes. Faz-se necessário instituir uma política de incentivo para que estes profissionais assumam o trabalho nestes locais e lá permaneçam.

Finalmente, sugerimos reciclagens e treinamentos para médicos generalistas, em patologias reumáticas, pois uma grande parte dos atendimentos feitos pelo especialistas poderia ser realizada por esses profissionais, além do estabelecimento de protocolos de encaminhamentos com 
a Classificação Internacional da Doença (CID-10) entre referências e contra-referências pactuadas, através do Sistema de Integração e Regulação do Município, qualificando

\section{REFERÊNCIAS}

1. Junqueira V: Observatório dos Direitos do Cidadão: acompanhamento e análise das políticas públicas da cidade de São Paulo, $2^{a}$ Edição revisada; Instituto Pólis/PUC, São Paulo, 2002.

2. Legislação e Normas pertinentes ao SUS. O que você precisa saber sobre o Sistema Unico de Saúde - vol 1. Associação Paulista de Medicina, I ${ }^{\underline{a}}$ Edição, São Paulo: 21-57, 2000.

3. Natalini, G:Princípios Básicos do SUS. O que você precisa saber sobre o Sistema Único de Saúde - vol 2. Associação Paulista de melhor o atendimento, aumentando a resolutividade e propiciando uma melhora na atenção a saúde aos clientes com patologia reumáticas.
Medicina, la Edição, São Paulo: 21-34, 2001.

4. Norma Operacional da Assistência à Saúde-NOAS-SUS 01/01. Regionalização da Assistência à Saúde: aprofundando a descentralização com eqüidade de acesso. Série A. Normas e Manuais Técnicos, no 116. Brasília - Distrito Federal - Ministério da Saúde - Secretaria de Assistência à Saúde: 9-26, 2001.

5. Portaria $n^{\circ} 1101 / \mathrm{GM}$, de 12/06/2002.

6. Estimativa da população exclusivamente usuária do SUS no Município de São Paulo. Documento da Secretaria Municipal da Saúde, São Paulo, 2004. 